\title{
Urine Proteomics and Biomarkers in Renal Disease
}

\author{
Min Jeong Kim Andrew H. Frankel Frederick W.K. Tam \\ Imperial College Kidney and Transplant Institute, Imperial College London, Hammersmith Hospital, London, UK
}

\section{Key Words}

Urine $\cdot$ Proteomics $\cdot$ Biomarker

\begin{abstract}
The application of urine proteomics is a useful approach to the study of the proteins involved in healthy and diseased kidneys and may provide a noninvasive approach to assess disease activity and to monitor clinical response in patients with renal diseases. This technique may provide an additional tool in clinical trials and for the assessment of prognosis for patients. Both soluble proteins and membrane-bound (exosomal) proteins may be studied, and multiple approaches are available. Discovery proteomics is an unbiased approach to detect novel proteins in urine samples. Mass spectrometry (MS) is often needed to identify specific protein fragments. Targeted proteomics often involves specific immunoassays or modified MS, which enables a hypothesisbased design. These approaches may be integrated. For example, specific proteins may be identified by the discovery approach or laboratory study of disease mechanisms. These proteins will then be studied further by targeted proteomics. In order to translate to clinical practice, the specific assays need vigorous validation by means of sufficiently statistically powered clinical trials.

Copyright $\odot 2011$ S. Karger AG, Basel
\end{abstract}

\section{Background}

Approximately $70 \%$ of the urinary proteomes in healthy individuals originate from the kidney and the urinary tract, and therefore urine samples are a valuable source, which can be utilized to identify biomarkers in patients with renal disease [1]. One of the early attempts to define the urinary proteome using liquid chromatography-tandem mass spectrometry (LC-MS/MS) was published in 2001 [2]. Since then, consistent efforts to identify efficient urinary biomarkers using different methods of proteomics have been made. Many studies on urinary proteomes in patients with different categories of renal disease have been conducted in the last decade, and the clinical application of some of the identified biomarkers may not be too far away from reality. A basic understanding of urinary proteomics will therefore be helpful not only for researchers but also for clinicians. The aim of this minireview is to provide concise information about currently available proteomic methods and the clinical application of urinary proteomics in renal disease.

\section{Introduction to Terminology}

The 'proteome' is the protein complement expressed by the genome. 'Proteomics' is the study of the proteome and can be further defined as the analysis of the spatial

Frederick W.K. Tam, MBBChir, PhD, FRCP

Imperial College Kidney and Transplant Institute, Hammersmith Hospital

Imperial College London

London W12 0NN (UK)

Tel. +44 208383 2354,E-Mail f.tam@imperial.ac.uk 
Table 1. Comparison of discovery and targeted urine proteomics

\begin{tabular}{lll}
\hline & Discovery & Targeted \\
\hline Methods & $\begin{array}{l}\text { gel electrophoresis } \\
\text { LC } \\
\text { capillary electrophoresis } \\
\text { MS }\end{array}$ & $\begin{array}{l}\text { immune-based assays (e.g. ELISA, } \\
\text { antibody arrays); modified MS }\end{array}$ \\
\hline Examples & $\begin{array}{l}\text { hepcidin in lupus nephritis; } \\
\text { fragments of collagen I in } \\
\text { diabetic nephropathy [12] }\end{array}$ & $\begin{array}{l}\text { CCL2 in proliferative glomerulone- } \\
\text { phritis [18, 19]; connective tissue growth } \\
\text { factor in diabetic nephropathy [20] }\end{array}$ \\
\hline Advantage & discovery of novel molecules & hypothesis based \\
\hline $\begin{array}{l}\text { Disadvantage } \\
\text { not hypothesis based }\end{array}$ & restricted to study of known proteins \\
\hline \multicolumn{1}{c}{ CCL2 = CC chemokine ligand 2 (also known as monocyte chemoattractant pro- } \\
tein-1).
\end{tabular}

and temporal expression of a subset (and ultimately a full set) of proteins in a defined biological system [3].

A major goal of proteomics in the clinical field is to identify biomarkers that can aid in the diagnosis and assessment of prognosis of different conditions. They may also be useful for monitoring therapeutic responses, optimization of treatment and guiding the choice of more appropriate therapies for each patient. Two different approaches in clinical proteomics can be termed 'discovery proteomics' and 'targeted proteomics' (table 1). In discovery proteomics, also referred to as 'fishing proteomics', the proteomes of the same biological samples from different groups of patients or healthy individuals are analyzed and compared to detect significant differences. The most common proteomic methods for this approach are various MS-based techniques. The advantage of this approach is the possibility to detect a novel candidate molecule as a new biomarker. The disadvantage, however, is that this approach is not hypothesis driven. In targeted proteomics, proteins to be investigated are defined beforehand, and appropriate methods, mostly immunology based, are applied for their detection. ELISA and protein microarrays are most commonly used. Efforts have also been made to adapt MS to detect a selective group of proteins [4]. Although this strategy is both powerful and useful to follow the changes in expression levels of specific proteins, it is not suitable for the discovery of a novel molecule involved in physiological processes.

Urine proteomics has traditionally focused on the study of soluble proteins. Recently, however, urinary exosomes have been receiving increasing attention as a new source of potential biomarkers. Exosomes are 40- to 100$\mathrm{nm}$ membrane vesicles of endocytic origin secreted by most cell types. They are found in body fluids and contain membrane proteins such as transporters and ion channels as well as cytosolic proteins. Urinary exosomes are a rich source of biomarkers because they are released from every segment of the nephron, including podocytes. After a purification process using several techniques, MS and Western blot are commonly used for further investigation [5]. In a small study, transcription factors were detected in the urinary exosomes from patients with acute kidney injury and focal segmental glomerulosclerosis [6].

\section{Overview of Currently Available Urine Proteomic Techniques}

\section{Methods Used for Discovery Proteomic Approach}

MS-based proteomic methods are in use for this approach, and each method consists of several steps. Initially, urine samples need to be handled by different methods such as centrifugal filtration, ultrafiltration, lyophilization or precipitation. Removal of albumin is sometimes required to improve the identification of lowabundance proteins. For certain MS techniques, protein digestion to peptides is necessary. The second step is the separation of proteins/peptides, and this can be achieved using 2-dimensional gel electrophoresis, LC or capillary electrophoresis (CE). The next step involves the mass spectrometric measurements of separated proteins/peptides. A mass spectrometer consists of an ion source, a mass analyzer that measures the mass-to-charge ratio $(\mathrm{m} / \mathrm{z})$ of the ionized analytes, and a detector that registers the number of ions at each $\mathrm{m} / \mathrm{z}$ value. Different ionization sources include matrix-assisted laser desorption/ioniza- 
tion, surface-enhanced laser desorption/ionization (SELDI) and electrospray ionization (ESI). Using the SELDI technique, the separation/fractionation of protein is redundant as this technique incorporates chromatographic and MS principles on a single platform. Different mass analyzers include ion trap, time-of flight (TOF), quadrupole, orbitrap and Fourier transform ion cyclotron resonance devices. In general, each method of protein separation (second step) can be combined with any MS techniques (third step), with the exception of SELDI, and some of them online. In the third step, a different ionization source again can be combined with a different mass analyzer, and sequential combination of two mass analyzers in tandem is also possible, enabling the sequencing of proteins/peptides. Therefore, theoretically, numerous combination possibilities exist; however, there are preferentially applied combinations in clinical proteomics. In the last step, the measured masses by MS need to be identified, and this is achieved by using computer programs that translate the known genome of the organism into proteins, then theoretically cut the proteins into peptides and calculate the absolute masses of the peptides from each protein. They then compare the measured masses of the peptides of the unknown protein to the theoretical peptide masses of each protein encoded in the genome. The results are statistically analyzed to find the best match [3, 7]. After all the above steps, a novel molecule can be identified as a potential biomarker, which can be studied further by targeted proteomics. In the following section, before moving over to targeted proteomic methods, commonly used MS-based discovery proteomic methods will be briefly reviewed.

\section{Two-Dimensional Gel Electrophoresis-Mass}

Spectrometry

After separation of proteins by 2-dimensional gel electrophoresis according to their isoelectric point and molecular weight, and 'in-gel' digestion of gel spots of interest by a protease, the produced peptides are commonly analyzed by matrix-assisted laser desorption/ionizationTOF. This technique allows the study of large molecules and enables the estimation of actual molecular weights and quantification of proteins [8]. This is the method of choice for the comparative analysis of unmodified medium-size or large proteins $(10-200 \mathrm{kDa})$ in the discovery phase of biomarker definition. However, there are several limitations such as low throughput, low reproducibility, high intensity of time and labor, and automation difficulties. Therefore, the method is not suitable for regular clinical application.

Urine Proteomics and Biomarkers
Liquid Chromatography-Mass Spectrometry

High-performance LC involves dissolving the protein mixture in buffer and pumping it through a series of columns to separate proteins according to different properties such as size exclusion, reverse phase, cation binding and affinity binding. Nano-LC has the highest sensitivity. The equipment is routinely configured for direct and continuous spraying of column effluents into an MS, which enables online detection of peptide ions and their fragmentation products. Tandem MS (ESI-quadrupole/ TOF or TOF/TOF) enables amino acid sequencing of selected peptides. This method is automated and offers a high sensitivity. Sequential separation using different media in two independent steps provides a multidimensional fractionation that can generate vast amounts of information. In a study of patients with Fanconi syndrome due to Dent's disease, more than 100 peptides were identified by this approach [9]. The variability on multidimensional separations, however, makes comparative analysis difficult, and the substantial amount of time required makes the technique unsuitable for clinical application. Furthermore, it is very sensitive to interference from lipid or detergent.

\section{Quantitative Comparison of Proteomes by Labeling}

Methods

Labeling methods have been developed to compare the proteomes between samples quantitatively. Isotope-coded affinity tags (ICAT) were used in earlier studies. The ICAT reagents label cysteine amino acid residues. The ICAT reagent containing ${ }^{13} \mathrm{C}$ can be used for one sample, the other sample is labeled with the same reagent containing ${ }^{12} \mathrm{C}$. After labeling, the samples are mixed and analyzed by LC-MS/MS. The relative quantities may be calculated from the ratio of the peptide fragments containing different carbon isotopes [8]. This method is limited to peptides with cysteine residues.

Improved methods based on the principle of ICAT have been developed. For example, isobaric tags for relative and absolute quantification may be used. These isobaric tags contain reporters with different molecular weights and a matched, balanced region to provide an overall identical molecular mass. The N-terminus and side chain amines of peptides are labeled with tags with different molecular mass reporters. Then, the labeled peptides from different urine samples may be mixed in equal proportion for analysis by LC-MS/MS. After fragmentation during MS, the reporters with different molecular masses are separated from the balance region and become distinguishable by MS. The relative quantity of 
Fig. 1. A schematic diagram showing the principle of SELDI-TOF MS to detect protein biomarkers. Urine samples are incubated with protein microchips. Activation of protein samples by laser results in ionization. The protein ions are accelerated in the presence of a high-voltage electrical field. The flight of protein ions is separated by the mass/charge ratio. The TOF of protein ions is detected to generate an MSTOF profile. Using this approach, protein peaks with specific mass/charge ratios were used as noninvasive urinary biomarkers to distinguish patients with active lupus nephritis from those in remission (part of the figure reproduced from Mosley et al. [11] with permission).

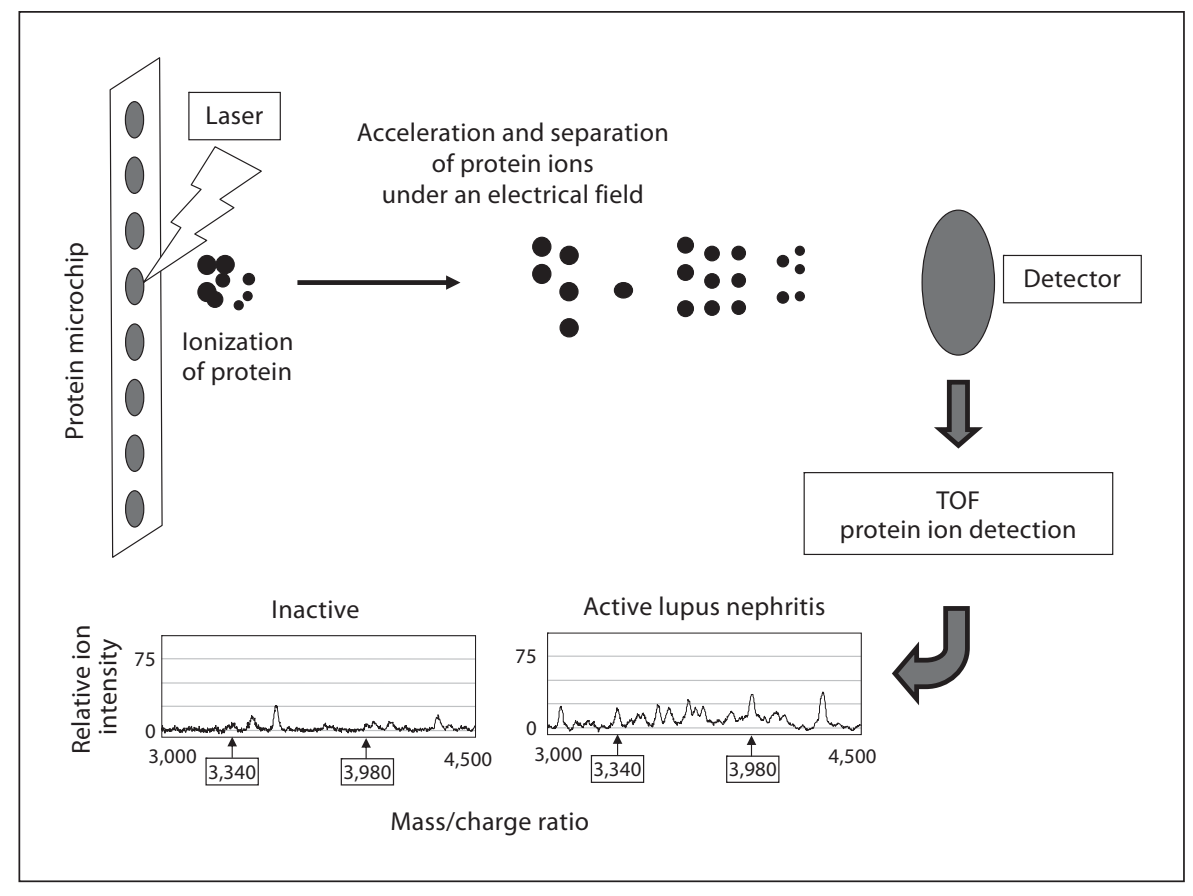

peptides from different samples will be quantified by the intensities of different reporter ions [10].

\section{Surface-Enhanced Laser Desorption/}

Ionization-Time-of-Flight

This technique incorporates chromatographic and MS principles on a single platform (fig. 1). An activated surface on a 'chip' binds proteins on the basis of their chemical and physical properties; unbound proteins are washed off. A subset of the proteome is thus selected, and the chip plugs directly into the MS for analysis. This is a useful high-throughput screening technique, and the relative speed and simplicity give it the potential for clinical use [11]. However, only a very small fraction of all proteins in a sample binds to the chip surface, and binding to the different chip surfaces varies depending on sample concentration, $\mathrm{pH}$, salt content and the presence of interfering compounds. Furthermore, it offers only a low resolution. Using this technique, our laboratory has detected a combination of urinary peptides which distinguishes patients with active lupus nephritis from those in remission, with greater sensitivity than traditional markers of disease activity including measurement of proteinuria, hematuria and serological markers. We found that these peptides were also useful for monitoring clinical responses and the detection of relapse [11]. Sequencing of the specific peptides may be difficult; however, Zhang et al. [12] over- came this and identified isoforms of hepcidin as novel urinary biomarkers of lupus nephritis by on-chip peptide sequencing using integrated tandem MS.

\section{Capillary Electrophoresis-Mass Spectrometry}

CE separates proteins in a silica capillary in a single step with a high resolution based on their migration through a gel in the electrical field. It is commonly combined with ESI-MS (ESI-TOF). This technique is robust and fast, uses inexpensive capillaries instead of expensive LC columns and generates comparable high-resolution data sets [13]. The data sets from individual analyses can be compiled to generate a typical proteome pattern that can be based on $>100$ individual analyses. This method is generally not suited for larger molecules $(>20 \mathrm{kDa})$; however, this is generally of less significance for urine samples as the urinary proteome contains a high percentage of low-molecular-weight proteins. Sequencing of protein can be achieved by directly interfacing CE with MS/ MS instruments, or by subsequent targeted sequencing using LC-MS/MS. CE-MS is therefore a good alternative to the commonly used proteomic technologies. In a study of patients with diabetes, a panel of 40 biomarkers was found to distinguish a subgroup of diabetic patients with different severities of nephropathy. It is interesting that many of these biomarkers are fragments of abundant proteins such as collagen type I. The mechanistic basis for 
and importance of using these abundant proteins as biomarkers need further investigation [14].

\section{Methods for Targeted Proteomic Approach}

While the MS-based techniques are applied for the detection of novel molecules (discovery proteomics), targeted proteomic methods are suitable for the validation of these molecules. They are mostly immunology-based and very powerful methods if the antibodies for the proteins under study are specific and readily available. Commonly used methods are ELISA, Western blot and protein microarray-based methods [15]. These methods are well established and widely applied in clinical diagnostics as well as research.

\section{ELISA and Western Blot}

The mechanism of both methods is the same. A specific antibody for the unknown amount of targeted molecule is applied to the surface so that binding of antibodies and targeted molecules can occur. This antibody is linked to an enzyme, and in the final step, a substance is added that the enzyme can convert to a detectable signal. The signal can be a color change, fluorescence or chemiluminescence. In Western blot, proteins in a sample need to be separated in a gel and blotted on a membrane before the detection procedure.

\section{Protein Microarray-Based Techniques}

A protein microarray provides an immunology-based technique for detecting multiple proteins. Specific antibodies or antigens are printed on a surface at separate locations in an ordered manner forming a microscopic array. The array surface can be a chip, bead, membrane or slide. The most common protein microarray is the antibody microarray, where antibodies are spotted onto the array surface and used as capture molecules to detect proteins from samples. Capture molecules commonly are antibodies; however, antigens can also be used if antibodies need to be detected in a sample. In our laboratory, we have used a membrane-bound antibody array to identify increased amounts of a profibrotic chemokine, CCL18, in the spent dialysis fluid from patients with long-term complications of peritoneal fibrosis [15] (fig. 2). The application of this technique to patient urine has been more difficult because of the lower concentrations of cytokines in urine. In this situation, it is more appropriate to use the more sensitive method of glass-based antibody arrays or Luminex bead arrays. With their extensive multiplexing capabilities, Luminex beads are becoming increasingly popular, and commercial kits are available for the detec-

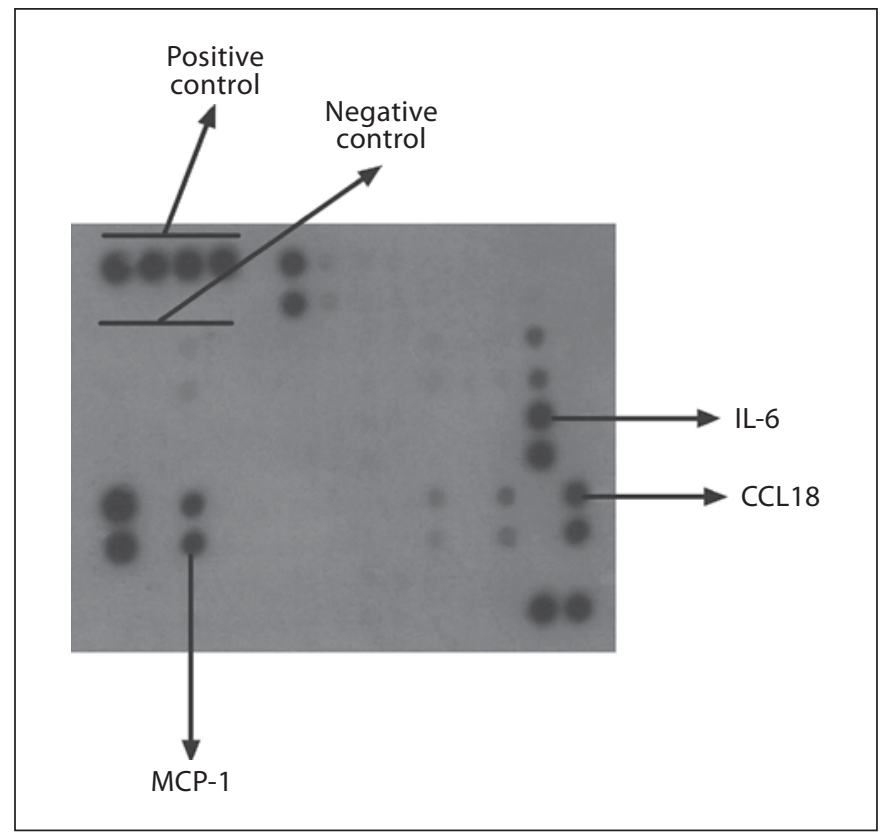

Fig. 2. Detection of cytokine proteins using a membrane-based multiplex cytokine antibody array. In this membrane-based array, 60 specific cytokines were tested in duplicate. Each dark spot represents a semiquantitative signal according to the cytokine concentration. Both positive and negative controls were included in the same array. In this experiment, high concentrations of CCL18, monocyte chemoattractant protein (MCP)-1 and interleukin (IL)- 6 were detected in the spent peritoneal dialysate from a long-term peritoneal dialysis patient. (Reproduced from Ahmad et al. [15] with permission).

tion of many different molecules, such as the multiplex cytokine array. These are very powerful methods as the technique is relatively simple and large numbers of samples can be investigated within a reasonable time. The limitations of these targeted proteomic methods are, however, the need for a specific probe for every protein to be analyzed. Furthermore, this kind of method may cause bias as the investigator chooses the set of antibodies to be used.

\section{Modified MS}

Recently, there have been developments in MS allowing the measurement of selected target proteins quantitatively, such as multiple reaction monitoring (MRM)based assays $[16,17]$. Specific antibodies are not needed for this technique. Efforts are being made to improve the reproducibility and throughput of these MRM-based assays in analyzing specific groups of proteins in biological fluids, so that they may be used in clinical situations. 


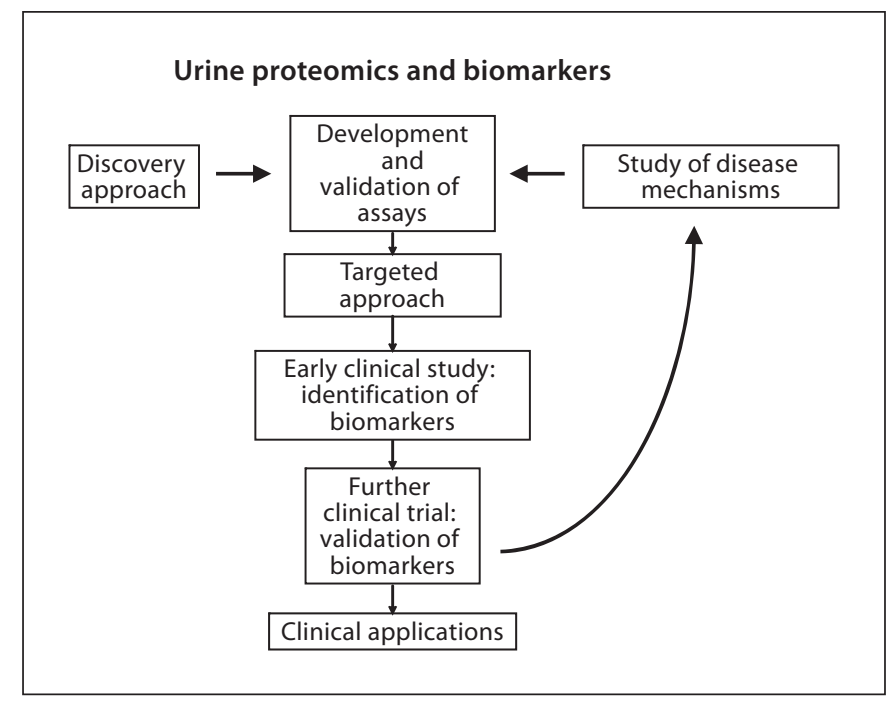

Fig. 3. A schematic diagram showing different approaches of urine proteomics leading to clinical studies, validation and applications.

\section{Application of Urine Proteomics: Workflow of Clinical Proteomics to Discover Urine Biomarkers}

The ideal sequence of urine proteomics to discover new biomarkers would be MS-based discovery of candidate molecules from small, well-defined patient populations, followed by a validation process with samples from large patient cohorts using immunology-based proteomic techniques and, subsequently, clinical application of biomarker assays. However, this is easier said than done, and modifications to this sequential approach are often made in reality. For example, the targeted candidate biomarker for a validation step can be derived from different sources such as in vitro and in vivo studies (fig. 3 ). These molecules can be investigated in samples from large patient groups using targeted proteomic methods. As they are already known to be involved in the pathogenesis of a disease from previous in vitro or in vivo research, the chance of validation is greater than for newly found molecules by MS discovery. For example, urinary CCL2 (also known as monocyte chemoattractant protein-1) was shown to be elevated in patients with proliferative glomerulonephritis $[18,19]$. In patients with diabetic nephropathy, we also found that urinary connective tissue growth factor and CCL2 are prognostic of progression of diabetic nephropathy [20].

Alternatively, it is possible to combine discovery and targeted proteomics using MS. For example, Quintana et al. [21] used LC-MS to discover that amounts of specific fragments of uromodulin and kininogen were lower in the urine of patients with chronic renal allograft dysfunction than in control subjects. These 5 specific protein fragment ions were measured using MRM-based LC-MS/ MS to validate the discovery.

It is critical to have adequate validation of any recently discovered biomarkers. This often involves validation of the reproducibility of the assays and clinical validation in large-scale clinical studies across multiple clinical centers (fig. 3). These are not simple tasks [22].

\section{Future Perspectives}

With continuous developments and improvements in proteomic methods and clinical trials regarding the validation of the candidate biomarkers, there will be more and more biomarkers or a battery of biomarkers available in the clinical field. These will aid in making diagnoses and prognoses and also in monitoring treatment responses, leading to an improvement in patient management. In the clinical research field, they can be used as surrogate markers, providing additional possibilities of assessing outcomes.

\section{Acknowledgments}

M.J.K. is in receipt of a research fellowship from the Swiss National Science Foundation and the Novartis Foundation. F.W.K.T. has been supported by the Diamond Fund from the Imperial College Healthcare Charity and the National Institute for Health Research (NIHR) Biomedical Research Centre funding scheme.

\section{Disclosure Statement}

F.W.K.T. has received research project grants from Roche Palo Alto, AstraZeneca Ltd. and Baxter Biosciences.

References $\quad{ }_{1}$ Decramer S, Gonzalez de Peredo A, Breuil B, Mischak H, Monsarrat B, Bascands JL, Schanstra JP: Urine in clinical proteomics. Mol Cell Proteomics 2008;7:1850-1862.

2 Spahr CS, Davis MT, McGinley MD, Robinson JH, Bures EJ, Beierle J, Mort J, Courchesne PL, Chen K, Wahl RC, Yu W, Luethy $\mathrm{R}$, Patterson SD: Towards defining the urinary proteome using liquid chromatography-tandem mass spectrometry. 1. Profiling an unfractionated tryptic digest. Proteomics 2001;1:93-107. 
3 Cutillas P, Burlingame A, Unwin R: Proteomic strategies and their application in studies of renal function. News Physiol Sci 2004;19:114-119.

4 Yocum AK, Chinnaiyan AM: Current affairs in quantitative targeted proteomics: multiple reaction monitoring-mass spectrometry. Brief Funct Genomic Proteomic 2009;8: 145-157.

5 Pisitkun T, Shen RF, Knepper MA: Identification and proteomic profiling of exosomes in human urine. Proc Natl Acad Sci USA 2004;101:13368-13373.

6 Zhou H, Cheruvanky A, Hu X, Matsumoto T, Hiramatsu N, Cho ME, Berger A, Leelahavanichkul A, Doi K, Chawla LS, Illei GG, Kopp JB, Balow JE, Austin HA 3rd, Yuen PS, Star RA: Urinary exosomal transcription factors, a new class of biomarkers for renal disease. Kidney Int 2008;74:613-621.

7 Aebersold R, Mann M: Mass spectrometrybased proteomics. Nature 2003;422:198-207.

$\checkmark 8$ Cutillas PR, Chalkley RJ, Hansen KC, Cramer R, Norden AG, Waterfield MD, Burlingame AL, Unwin RJ: The urinary proteome in Fanconi syndrome implies specificity in the reabsorption of proteins by renal proximal tubule cells. Am J Physiol Renal Physiol 2004;287:F353-F364.

$\checkmark 9$ Cutillas PR, Norden AG, Cramer R, Burlingame AL, Unwin RJ: Detection and analysis of urinary peptides by on-line liquid chromatography and mass spectrometry: application to patients with renal Fanconi syndrome. Clin Sci (Lond) 2003;104:483-490.

-10 Akkina SK, Zhang Y, Nelsestuen GL, Oetting WS, Ibrahim HN: Temporal stability of the urinary proteome after kidney transplant: more sensitive than protein composition? J Proteome Res 2009;8:94-103.
11 Mosley K, Tam FWK, Edwards RJ, Crozier J, Pusey CD, Lightstone L: Urinary proteomic profiles distinguish between active and inactive lupus nephritis. Rheumatology (Oxford) 2006;45:1497-1504.

12 Zhang X, Jin M, Wu H, Nadasdy T, Nadasdy G, Harris N, Green-Church K, Nagaraja H, Birmingham DJ, Yu CY, Hebert LA, Rovin $\mathrm{BH}$ : Biomarkers of lupus nephritis determined by serial urine proteomics. Kidney Int 2008;74:799-807.

13 Metzger J, Schanstra JP, Mischak H: Capillary electrophoresis-mass spectrometry in urinary proteome analysis: current applications and future developments. Anal Bioanal Chem 2009;393:1431-1442.

14 Rossing K, Mischak H, Dakna M, Zurbig P, Novak J, Julian BA, Good DM, Coon JJ, Tarnow L, Rossing P: Urinary proteomics in diabetes and CKD. J Am Soc Nephrol 2008;19: 1283-1290.

15 Ahmad S, North BV, Qureshi A, Malik A, Bhangal G, Tarzi RM, Brown EA, Tam FWK: CCL18 in peritoneal dialysis patients and encapsulating peritoneal sclerosis. Eur J Clin Invest 2010;40:1067-1073.

16 Addona TA, Abbatiello SE, Schilling B, Skates SJ, Mani DR, Bunk DM, Spiegelman CH, Zimmerman LJ, Ham AJ, Keshishian H, Hall SC, Allen S, Blackman RK, Borchers $\mathrm{CH}$, Buck C, Cardasis HL, Cusack MP, Dodder NG, Gibson BW, Held JM, Hiltke T, Jackson A, Johansen EB, Kinsinger CR, Li J, Mesri M, Neubert TA, Niles RK, Pulsipher TC, Ransohoff D, Rodriguez H, Rudnick PA, Smith D, Tabb DL, Tegeler TJ, Variyath AM, Vega-Montoto LJ, Wahlander A, Waldemarson S, Wang M, Whiteaker JR, Zhao L, Anderson NL, Fisher SJ, Liebler DC, Paulovich AG, Regnier FE, Tempst P, Carr SA: Multisite assessment of the precision and reproducibility of multiple reaction monitoringbased measurements of proteins in plasma. Nat Biotechnol 2009;27:633-641.
17 Kiyonami R, Schoen A, Prakash A, Peterman S, Zabrouskov V, Picotti P, Aebersold R, Huhmer A, Domon B: Increased selectivity, analytical precision, and throughput in targeted proteomics. Mol Cell Proteomics 2010, E-pub ahead of print.

18 Tam FWK, Sanders JS, George A, Hammad T, Miller C, Dougan T, Cook HT, Kallenberg CGM, Gaskin G, Levy JB, Pusey CD: Urinary monocyte chemoattractant protein-1 (MCP1 ) is a marker of active renal vasculitis. Nephrol Dial Transplant 2004;19:2761-2768.

19 Rovin BH, Song H, Birmingham DJ, Hebert LA, Yu CY, Nagaraja HN: Urine chemokines as biomarkers of human systemic lupus erythematosus activity. J Am Soc Nephrol 2005; 16:467-473

20 Tam FWK, Riser BL, Meeran K, Rambow J, Pusey CD, Frankel AH: Urinary monocyte chemoattractant protein-1 (MCP-1) and connective tissue growth factor (CCN2) as prognostic markers for progression of diabetic nephropathy. Cytokine 2009;47:37-42.

-21 Quintana LF, Campistol JM, Alcolea MP, Banon-Maneus E, Sol-Gonzalez A, Cutillas PR: Application of label-free quantitative peptidomics for the identification of urinary biomarkers of kidney chronic allograft dysfunction. Mol Cell Proteomics 2009;8:16581673.

22 Knepper MA: Common sense approaches to urinary biomarker study design. J Am Soc Nephrol 2009;20:1175-1178. 